\title{
Classification of patients with sepsis according to blood genomic endotype: a prospective cohort study
}

\author{
Brendon P Scicluna, Lonneke A van Vught, Aeilko H Zwinderman, Maryse A Wiewel, Emma E Davenport, Katie L Burnham, Peter Nürnberg, \\ Marcus J Schultz, Janneke Horn, Olaf L Cremer, Marc J Bonten, Charles J Hinds, Hector R Wong, Julian C Knight, Tom van der Poll, on behalf of the \\ MARS consortium*
}

\section{Summary}

Background Host responses during sepsis are highly heterogeneous, which hampers the identification of patients at high risk of mortality and their selection for targeted therapies. In this study, we aimed to identify biologically relevant molecular endotypes in patients with sepsis.

Methods This was a prospective observational cohort study that included consecutive patients admitted for sepsis to two intensive care units (ICUs) in the Netherlands between Jan 1, 2011, and July 20, 2012 (discovery and first validation cohorts) and patients admitted with sepsis due to community-acquired pneumonia to 29 ICUs in the UK (second validation cohort). We generated genome-wide blood gene expression profiles from admission samples and analysed them by unsupervised consensus clustering and machine learning. The primary objective of this study was to establish endotypes for patients with sepsis, and assess the association of these endotypes with clinical traits and survival outcomes. We also established candidate biomarkers for the endotypes to allow identification of patient endotypes in clinical practice.

Findings The discovery cohort had 306 patients, the first validation cohort had 216, and the second validation cohort had 265 patients. Four molecular endotypes for sepsis, designated Mars1-4, were identified in the discovery cohort, and were associated with 28-day mortality (log-rank $\mathrm{p}=\mathbf{0}$.022). In the discovery cohort, the worst outcome was found for patients classified as having a Mars1 endotype, and at 28 days, $35(39 \%)$ of 90 people with a Mars1 endotype had died (hazard ratio [HR] vs all other endotypes 1.86 [95\% CI 1.21-2.86]; p=0.0045), compared with $23(22 \%)$ of 105 people with a Mars2 endotype (HR 0.64 [0.40-1.04]; p=0 061), 16 (23\%) of 71 people with a Mars3

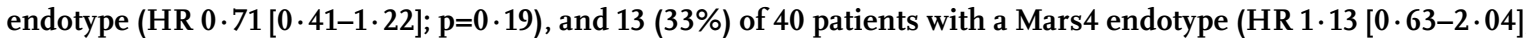
$\mathrm{p}=0.69)$. Analysis of the net reclassification improvement using a combined clinical and endotype model significantly improved risk prediction to $0.33(0.09-0.58 ; \mathrm{p}=0.008)$. A 140 -gene expression signature reliably stratified patients with sepsis to the four endotypes in both the first and second validation cohorts. Only Mars1 was consistently significantly associated with 28-day mortality across the cohorts. To facilitate possible clinical use, a biomarker was derived for each endotype; BPGM and TAP2 reliably identified patients with a Mars1 endotype.

Interpretation This study provides a method for the molecular classification of patients with sepsis to four different endotypes upon ICU admission. Detection of sepsis endotypes might assist in providing personalised patient management and in selection for trials.

Funding Center for Translational Molecular Medicine, Netherlands.

\section{Introduction}

Sepsis remains a remarkable adversary to medicine, characterised by poor prognosis and high mortality rates. $^{1,2}$ Despite the burden on patients, their families, and the health-care system, treatment remains mainly non-curative. ${ }^{1}$ Unrecognised population substructures and the heterogeneity in the host response complicate the identification of high-risk patients who would benefit from specific adjuvant therapy. ${ }^{3}$

Blood transcriptional profiling has led to substantial advances in sepsis treatment. ${ }^{4}$ Although promising new diagnostic biomarkers have emerged from the application of blood genomics to sepsis, ${ }^{5-7}$ patient selection for interventional trials and prediction of patient outcomes in sepsis continue to be driven by clinical signs. Although supervised analyses of patients with sepsis who are discordant for survival (ie, those who survive and those who do not) have identified candidate protein and gene expression prognostic markers, ${ }^{8,9}$ substantial heterogeneity remains unexplained. Unsupervised learning coupled with adequate validation metrics have been successfully applied in the field of oncogenomics to analyse the tumour heterogeneity in patients with cancer, ${ }^{10,11}$ which revealed important patient endotypes that would have otherwise remained unknown. A comprehensive assessment of the heterogeneity in the adult host response to all-cause sepsis in consecutive intensive care unit (ICU) admissions has not previously been done.

The primary objective of this study was to identify subgroups (endotypes) of patients with sepsis on the basis of whole-blood RNA expression profiles. To achieve this aim, we used unsupervised consensus clustering and machine learning in a discovery cohort of patients
Lancet Respir Med 2017

Published Online August 29, 2017 http://dx.doi.org/10.1016/ S2213-2600(17)30294-1

See Online/Comment http://dx.doi.org/10.1016/ S2213-2600(17)30297-7

*Members listed in the appendix Center for Experimental Molecular Medicine (B P Scicluna PhD, $L A$ van Vught MD, $M$ A Wiewel MD, Prof T van der Poll MD), Department of Clinical Epidemiology, Biostatistics and Bioinformatics (B P Scicluna,

Prof A H Zwinderman PhD), Department of Intensive Care Medicine (Prof M J Schultz MD J Horn MD), and Division of Infectious Diseases (ProfT van der Poll), Academic Medical Center, Amsterdam, Netherlands; Wellcome Trust Centre for Human Genetics, University of Oxford, Oxford, UK (E E Davenport DPhil,

KL Burnham MGen, Prof J ( Knight DPhil); Cologne Center for Genomics (Prof P Nürnberg PhD), Cologne Excellence Cluster on Cellular Stress Responses in Aging-Associated Diseases (Prof P Nürnberg), and Center for Molecular Medicine Cologne (Prof P Nürnberg), University of Cologne, Cologne, Germany; Department of Intensive Care Medicine (O L Cremer MD) and Department of Medical Microbiology (Prof M J Bonten MD), University Medical Center Utrecht, Utrecht, Netherlands; William Harvey Research Institute, Barts and The London School of Medicine Queen Mary University, London, UK (Prof C J Hinds MD); Division of Critical Care Medicine, Cincinnati Children's Hospital Medical Center and Cincinnati Children's Research 
Foundation, Cincinnati, $\mathrm{OH}$, USA (Prof H R Wong MD); and Department of Pediatrics, University of Cincinnati College of Medicine, Cincinnati, $\mathrm{OH}, \mathrm{USA}$ (Prof HRWong)

Correspondence to: Dr Brendon P Scicluna, Center for Experimental Molecular Medicine and Department of

Clinical Epidemiology, Biostatistics and Bioinformatics, Academic Medical Center, University of Amsterdam, Amsterdam 1105AZ, Netherlands b.scicluna@amc.uva.nl See Online for appendix

\section{Research in context}

\section{Evidence before this study}

Pronounced heterogeneity in the host response to sepsis complicates the identification of patients who are critically ill and at high risk of mortality and those who would benefit from specific adjuvant therapy. We searched for blood genomic studies of critically ill patients with sepsis published in any language before Jan 31, 2017, using the following search terms: ("sepsis" OR "severe sepsis" OR "septic shock") AND ("genomics" OR "gene expression profiling" OR "microarray") AND (subtype OR endotype OR subclassification OR cluster OR subgroup OR prospective cohort). We subsequently added "patient" to a second search field. We identified 38 studies of peripheral blood leucocytes in patients. Of these 38 studies, three applied genome-wide blood transcriptional profiling coupled with clustering techniques showing the existence of population substructures, also known as endotypes, in paediatric patients with sepsis, adult patients with sepsis, and patients with sepsis caused by community-acquired pneumonia.

\section{Added value of this study}

To the best of our knowledge, ours is the first study to comprehensively investigate the occurrence of patient endotypes in a consecutively enrolled population of patients with all-cause sepsis and validate the results in multiple independent datasets.
We identified four blood gene expression endotypes in patients from the UK and the Netherlands, one of which (Mars1) was consistently significantly associated with acute (28-day) and late (1-year) mortality. Furthermore, combining Acute Physiology and Chronic Health Evaluation IV scores and endotype significantly improved patient risk stratification. The four endotypes could not be predicted by demographic or clinical covariates. We also showed that the blood transcriptomes of these four endotypes had distinct host response signatures, including endotypes attuned to immunosuppression, hyperinflammation, or adaptive immune functions, showing great potential for more targeted patient management and clinical trial design.

\section{Implications of all the available evidence}

The substantial heterogeneity in the host response to sepsis has hindered patient management and therapeutic discoveries. Our study and others have shown that using the concepts of unsupervised blood genomic analysis, patients can be classified into molecular endotypes with important prognostic and pathophysiological value. The distinct host response signatures between the four endotypes have important implications that include development of precision therapeutics and practices of clinical trial design. admitted to the ICU with sepsis, and subsequently tested the robustness of our results across two independent validation cohorts from different hospitals. Furthermore, we established candidate blood genomic biomarkers for sepsis endotype classification and assessed them in our cohorts.

\section{Methods \\ Study design and participants}

This study was done within the wider context of the Molecular Diagnosis and Risk Stratification of Sepsis (MARS) project (NCT01905033), a prospective observational cohort study in the mixed ICUs of two tertiary teaching hospitals (Academic Medical Center in Amsterdam, Netherlands, and University Medical Center in Utrecht, Netherlands). ${ }^{5,12,13}$

All patients older than 18 years admitted to the two ICUs between Jan 1, 2011, and July 20, 2012, with an expected length of stay longer than $24 \mathrm{~h}$ were included via an opt-out method approved by the medical ethical committees of the participating hospitals., ${ }^{5,1213}$ For every patient admitted, the plausibility of an infection was assessed retrospectively using a four-point scale (ascending from none, possible, probable, to definite) using the Centers for Disease Control and Prevention ${ }^{14}$ and International Sepsis Forum consensus definitions ${ }^{15}$ as previously described. ${ }^{12}$ Our study comprised consecutive patients admitted to the ICU with sepsis defined as probable or definite infection (for site-specific criteria, see Klein Klouwenberg and colleagues ${ }^{12}$ ), accompanied by at least one additional general, inflammatory, haemodynamic, organ dysfunction, or tissue perfusion variable described in the report from the 2001 International Sepsis Definitions Conference (appendix p 6). ${ }^{16}$ Patients admitted to hospital in Amsterdam were used as the discovery cohort and those admitted to hospital in Utrecht were the first validation cohort. Healthy controls were also enrolled from the Academic Medical Center and De Drecht home for the elderly (both Amsterdam, Netherlands) after providing written informed consent. Criteria for inclusion in the healthy control group was age of at least 18 years. The second validation cohort was from the UK Genomic Advances in Sepsis (GAinS) study ${ }^{17}$ of adult patients with sepsis resulting from community-acquired pneumonia. Additionally, paediatric patients with sepsis from a prospective observational study ${ }^{18}$ of children aged 10 years or younger admitted to multiple paediatric ICUs in the USA were used as a comparative cohort (for GAinS and paediatric cohort inclusion criteria, see appendix $\mathrm{p} 3$ ).

\section{Procedures}

From patients enrolled in MARS, we collected blood specifically for this study in PAXgene blood RNA tubes (Becton-Dickinson, Breda, Netherlands) within $24 \mathrm{~h}$ of ICU admission. We also collected blood from the healthy controls in PAXgene blood RNA tubes. We generated 
gene expression profiles of patients and healthy participants for this study using Human Genome U219 96-array plates and the GeneTitan instrument (Affymetrix; Santa Clara, CA, USA) as described., ${ }^{5,13}$ MARS gene expression data are available in the Gene Expression Omnibus, accession number GSE65682. We obtained publicly available gene expression data of UK GAinS (ArrayExpress accession number E-MTAB-4421) generated by Illumina Human-HT-12 version 4 Expression BeadChips (San Diego, CA, USA). ${ }^{17}$ We also obtained publicly available gene expression data of paediatric patients with sepsis (GSE13904) that were generated by the Affymetrix Human Genome U133 Plus 2.0 Array. ${ }^{18}$

We analysed gene expression data from the discovery cohort using a previously developed unsupervised consensus clustering method (appendix $\mathrm{p}$ 3). ${ }^{19-21}$ For endotype discovery (appendix p 3), we ranked probes by median absolute deviation across 306 patient samples (discovery cohort). We selected and analysed the top 5000 ranked probes using the consensus clustering method. ${ }^{19,20}$ We selected the agglomerative hierarchical clustering algorithm on 1 minus Pearson correlation distances, 99\% item (sample) resampling, 1000 iterations, and cluster range $k=2-12$. To estimate $k$ (number of endotypes), we combined cumulative distribution functions, ${ }^{19,20}$ silhouette width analysis ${ }^{22}$ available in the cluster package, ${ }^{23}$ and cophenetic distance correlation analysis to assess clustering stability. ${ }^{24}$ To construct the $k$ endotype classifier, we selected patient samples with positive silhouette widths, representing core patients per endotype. ${ }^{19,22}$ We subsequently ranked the 5000 probes by non-parametric significance (Kruskal-Wallis rank sum test). We filtered 2994 unique gene probes by selecting for the highest significance. Using a random forest classifier ${ }^{25}$ (supervised classification with high dimensional data methods), ${ }^{26}$ we assessed sepsis endotype classification with ten-fold cross-validation of stepwise increments in gene numbers. We settled on the number of genes that yielded a cross-validation misclassification error rate of less than $10 \%$. We then used the sepsis endotype classifier gene set to do random forest prediction of endotypes in the validation cohorts. We used the healthy individuals solely for comparing gene expression profiles with those of patients in the MARS project classified into endotypes (herein termed Mars endotypes). See appendix (pp 3-4, 14) for further description of clustering methodology and differential gene expression analysis. Endotype

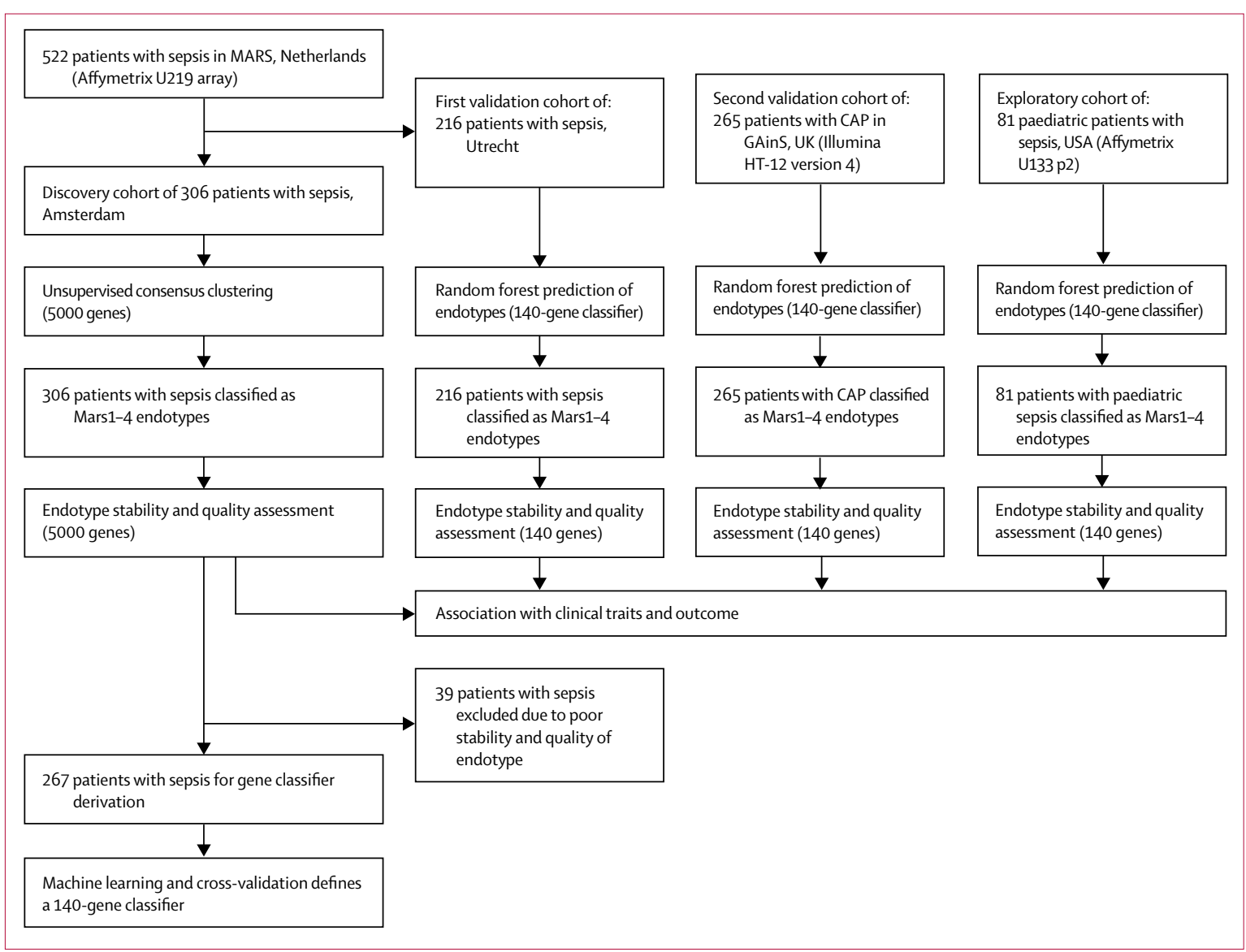

Figure 1: Patient cohorts, samples, and analysis

CAP=community-acquired pneumonia. GAinS=Genomic Advances in Sepsis cohort. MARS=Molecular Diagnosis and Risk Stratification of Sepsis cohort. 


\begin{tabular}{|c|c|c|c|}
\hline & $\begin{array}{l}\text { Discovery cohort, } \\
\text { Amsterdam, Netherlands } \\
(\mathrm{N}=306)\end{array}$ & $\begin{array}{l}\text { First validation cohort, } \\
\text { Utrecht, Netherlands } \\
\text { ( } \mathrm{N}=216)\end{array}$ & $\begin{array}{l}\text { Second validation } \\
\text { cohort, UK }(\mathrm{N}=265)\end{array}$ \\
\hline \multicolumn{4}{|l|}{ Demographics } \\
\hline Male sex & $166(54 \%)$ & $131(61 \%)$ & $145(55 \%)$ \\
\hline Age, years & $63(52-72)$ & $63(55-71)$ & $64(52-75)$ \\
\hline White ethnicity & $241(79 \%)$ & $208(96 \%)$ & .. \\
\hline \multicolumn{4}{|l|}{ Chronic comorbidity } \\
\hline None & $124(41 \%)$ & $37(17 \%)$ & .. \\
\hline $\begin{array}{l}\text { Cardiovascular } \\
\text { compromise }\end{array}$ & $53(17 \%)$ & $55(25 \%)$ & $118(45 \%)$ \\
\hline COPD & $39(13 \%)$ & $30(14 \%)$ & $62(23 \%)$ \\
\hline Diabetes & $54(18 \%)$ & $45(21 \%)$ & $51(19 \%)$ \\
\hline Hypertension & $66(22 \%)$ & $76(35 \%)$ & .. \\
\hline Malignancy & $36(12 \%)$ & $74(34 \%)$ & $17(6 \%)$ \\
\hline Renal insufficiency & $41(13 \%)$ & $36(17 \%)$ & $28(11 \%)$ \\
\hline Respiratory insufficiency & $47(15 \%)$ & $33(15 \%)$ & $128(48 \%)$ \\
\hline $\begin{array}{l}\text { Charlson comorbidity } \\
\text { index }\end{array}$ & $4(2-5)$ & $5(3-6)$ & .. \\
\hline Lung & $130(42 \%)$ & $96(44 \%)$ & $265(100 \%)$ \\
\hline Abdominal & $79(26 \%)$ & $51(24 \%)$ & .. \\
\hline Urinary & $25(8 \%)$ & $24(11 \%)$ & .. \\
\hline Skin & $24(8 \%)$ & $6(3 \%)$ & .. \\
\hline Cardiovascular & $11(4 \%)$ & $7(3 \%)$ & \\
\hline CNS & $5(2 \%)$ & $4(2 \%)$ &.. \\
\hline Other* & $32(10 \%)$ & $28(13 \%)$ & .. \\
\hline \multicolumn{4}{|c|}{ Severity at time of admission to ICU } \\
\hline APACHE score & $77(60-97) \dagger$ & $85(69-103) \dagger$ & $18(14-22) \ddagger$ \\
\hline SOFA score & $8(6-10)$ & $6(4-9)$ & $6(4-9)$ \\
\hline Shock & $108(35 \%)$ & $73(34 \%)$ & $79(30 \%)$ \\
\hline Acute kidney injury & $132(43 \%)$ & $55(25 \%)$ & $52(20 \%)$ \\
\hline Acute lung injury & $101(33 \%)$ & $52(24 \%)$ & .. \\
\hline \multicolumn{4}{|l|}{ Outcome } \\
\hline Length of stay, days & $4(2-9)$ & $6(2-12)$ & $7(4-15)$ \\
\hline ICU mortality & $58(19 \%)$ & $37(17 \%)$ & $49(18 \%)$ \\
\hline Hospital mortality & $100(33 \%)$ & $57(26 \%)$ & $68(26 \%)$ \\
\hline 14-day mortality & $73(24 \%)$ & $36(17 \%)$ & $40(15 \%)$ \\
\hline 28-day mortality & $87(28 \%)$ & $46(21 \%)$ & $56(21 \%)$ \\
\hline 90-day mortality & $113(37 \%)$ & $70(32 \%)$ & .. \\
\hline 1-year mortality & $139(45 \%)$ & $97(45 \%)$ & .. \\
\hline
\end{tabular}

Data are $\mathrm{n}(\%)$ or median (IQR). . -means data were unavailable. COPD=chronic obstructive pulmonary disease. ICU=intensive care unit. APACHE=Acute Physiology And Chronic Health Evaluation. SOFA=sequential organ failure assessment. *Includes bone and joint infection, endocarditis, mediastinitis, myocarditis, ear infection, oral infection, pharyngitis, postoperative wound infection, and lung abscess. †APACHE IV score. ¥APACHE II score.

Table: Baseline characteristics and mortality of patients in the discovery and validation cohorts

For the R project software see http://www.R-project.org/ biomarkers were assessed ${ }^{5,6}$ and differential gene expression and ingenuity pathway analysis was done using previously described methods (appendix pp 3-4).

\section{Outcomes}

The primary objective of this study was to establish endotypes for patients with sepsis, and assess the association of these endotypes with clinical traits and survival outcomes. We also investigated biomarkers for the endotypes, which could allow identification of patient endotypes in clinical practice.

\section{Statistical analysis}

Statistical analysis was done using the $\mathrm{R}$ statistical computing environment (version 3.1.2). The Cramér's $\mathrm{V}$ measure of effect size was used for a $\chi^{2}$ goodness-offit test. Correlation analysis of continuous data was done using Spearman's rank correlation coefficient. Survival analysis was done by Kaplan-Meier estimation (log-rank test) and Cox proportional hazards regression implemented in the survival method ( $\mathrm{R}$ package, version 2.37). Hazard ratios (HRs) and $95 \%$ CIs were calculated for each endotype with reference to all other endotypes. Net reclassification improvement was assessed by means of a continuous model using the predictABEL method (version 1.2-2). ${ }^{27}$ One model encompassed only Acute Physiology and Chronic Health Evaluation (APACHE) IV scores $^{28}$ (clinical), whereas a second model encompassed both APACHE IV scores and sepsis endotype stratification (clinical plus molecular). Results were considered significant at $\mathrm{p}<0 \cdot 05$.

\section{Role of the funding source}

The funders of the study had no role in the study design, data collection, data analysis, data interpretation, or writing of the report. The corresponding author had full access to all the data in the study and had final responsibility for the decision to submit for publication.

\section{Results}

From the MARS project, we included 306 patients in our discovery cohort and 216 in the first validation cohort (figure 1, table). We included 265 patients from the GAinS study ${ }^{17}$ as the second validation cohort. We also enrolled 42 healthy controls (median age 35 years [IQR 30-63]; 24 [57\%] of 42 were men) between Jan 1, 2011, and Dec 22, 2012.

Patient cohorts were characterised by various comorbidities, organ failure, and shock on admission in about a third of the patients (table). These baseline patient characteristics exemplify the marked heterogeneity in the clinical presentation of critically ill patients with sepsis. ${ }^{1.3}$

Considering cluster (endotype) quality and stability, ${ }^{22,24}$ we reached a consensus in partitioning at four molecular endotypes, designated Mars1-4 (appendix p 15) in the discovery cohort. In the discovery and first validation cohorts, most patients were white (table) and we found no association between ethnicity and endotype (appendix pp 7-10). In the discovery cohort, patients who had positive silhouette widths ${ }^{22}$ (81 [90\%] of 90 patients with the Mars1 endotype, 94 [90\%] of 105 with Mars2, 63 [89\%] of 71 with Mars3, and 29 [73\%] of 40 with Mars4), indicative of their high intra-endotype similarity, ${ }^{1,2,1,22}$ were used for gene classifier derivation (39 patients without 
positive silhouette widths were excluded; figure 1). We identified 140 genes that classified the Mars1-4 endotypes (appendix pp 15, 22-25).

In the discovery cohort, sepsis endotypes did not show an association with any comorbidities, APACHE IV score, or acute lung injury at admission to the ICU (appendix pp 7-8); however, septic shock prevalence and sequential organ failure assessment (SOFA) scores $^{29}$ were significantly associated with endotype classification (figure 2A, B). The estimated effect size (Cramér's V) of septic shock was $0 \cdot 23$, indicating a moderate association. Kaplan-Meier analysis showed an association between endotype and 28 -day mortality (log-rank $\mathrm{p}=0 \cdot 022$; figure $2 \mathrm{C}$ ). The worst survival outcome at 28 days was seen for patients with the Mars1 endotype (HRs given vs all other endotypes grouped as one): mortality occurred in 35 (39\%) of 90 people with the Mars1 endotype (HR 1.86 [95\% CI 1.21-2.86]; $\mathrm{p}=0.0045), 23$ (22\%) of 105 with Mars 2 (HR 0.64 [0.4-1.04]; $\mathrm{p}=0 \cdot 061), 16$ (23\%) of 71 with Mars3 (HR 0.71 [0.41-1.22]; $\mathrm{p}=0 \cdot 19)$, and 13 (33\%) of 40 with Mars4 (HR 1.13 [0.63-2.04]; $\mathrm{p}=0.69$ ). The multivariate HR (incorporating Charlson comorbidity indices) for death within 28 days of Mars1 classification was 1.79 (95\% CI 1.16-2.75; $\mathrm{p}=0 \cdot 0084)$. The patients with Mars1 endotype had the worst survival outcome at 1 year of patient follow-up (log-rank $\mathrm{p}=0 \cdot 023$; appendix $\mathrm{p} 16$ ).

To test whether the combination of a molecular and clinical scoring system might be of benefit to patient risk stratification, we assessed the net reclassification improvement and integrated discrimination improvement ${ }^{27,30}$ using a combined APACHE IV score (clinical) and sepsis endotype classification (molecular) model. This clinicomolecular model significantly improved 28-day mortality risk prediction compared with 28-day mortality risk prediction by APACHE IV scores alone (net reclassification improvement, continuous 0.33 [95\% CI 0.09-0.58], $\mathrm{p}=0 \cdot 008$; integrated discrimination improvement $0 \cdot 015[0 \cdot 0002-0 \cdot 03], \mathrm{p}=0 \cdot 047$; appendix $\mathrm{p}$ 16). The Hosmer-Lemeshow test showed proper model calibration (appendix p 16).

In the first validation cohort of patients with all-cause sepsis $(n=216)$, applying the 140-gene classifier clearly identified four sepsis endotypes (appendix $p$ 17). Consistent with the discovery cohort, SOFA scores and septic shock were significantly associated with sepsis endotype (figure $3 \mathrm{~A}, \mathrm{~B}$ ), with a moderate association with septic shock (Cramér's V=0 -29). APACHE IV scores were not associated with endotype classification, whereas Kaplan-Meier analysis showed a significant association with 28-day mortality (figure 3C; appendix pp 9, 17). The highest mortality was also found for patients classified as Mars1 in this cohort, and 19 (32\%) of 60 patients had died at 28 days (HR 1.97 [95\% CI 1.11-3.54]; p=0.024 vs Mars2-4). However, risk stratification by Mars2-4 showed more variance than in the discovery cohort, especially in Mars4. At 28 days, 18 (23\%) of 79 patients with the Mars2 endotype (HR 1.12 [0.62-2.03]; $\mathrm{p}=0 \cdot 69)$, eight $(14 \%)$ of
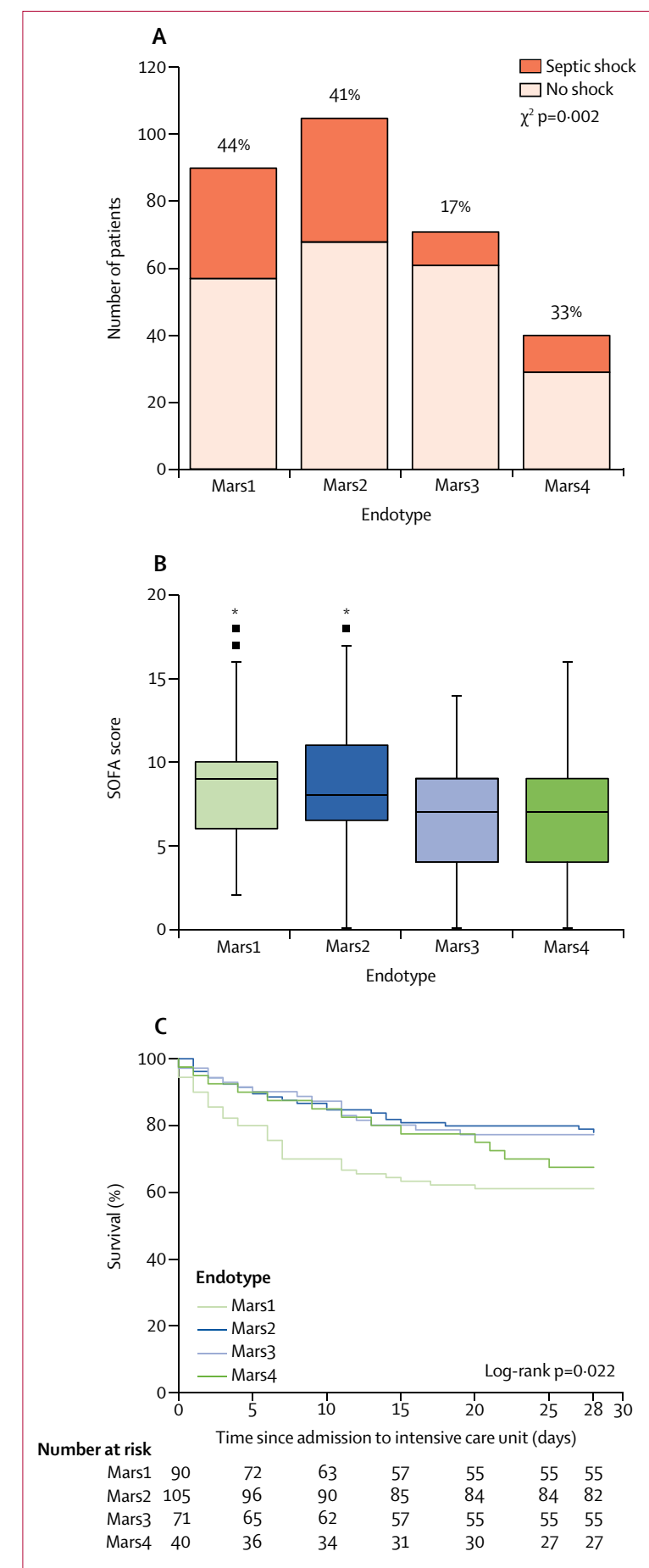

Figure 2: Association with clinical characteristics and outcome in the discovery cohort

Endotypes were evaluated for their association to clinical severity indices, septic shock ( $A$; the proportions of patients with septic shock within each endotype are shown at the top of each bar), SOFA scores (B; black horizontal line shows median score, top and bottom of box shows upper and lower quartiles, whiskers show extremes, and squares show outliers), and Kaplan-Meier survival analysis up to day 28 (C). SOFA=sequential organ failure assessment. *Dunn's post-hoc test $p<0.01$ vs Mars3. 


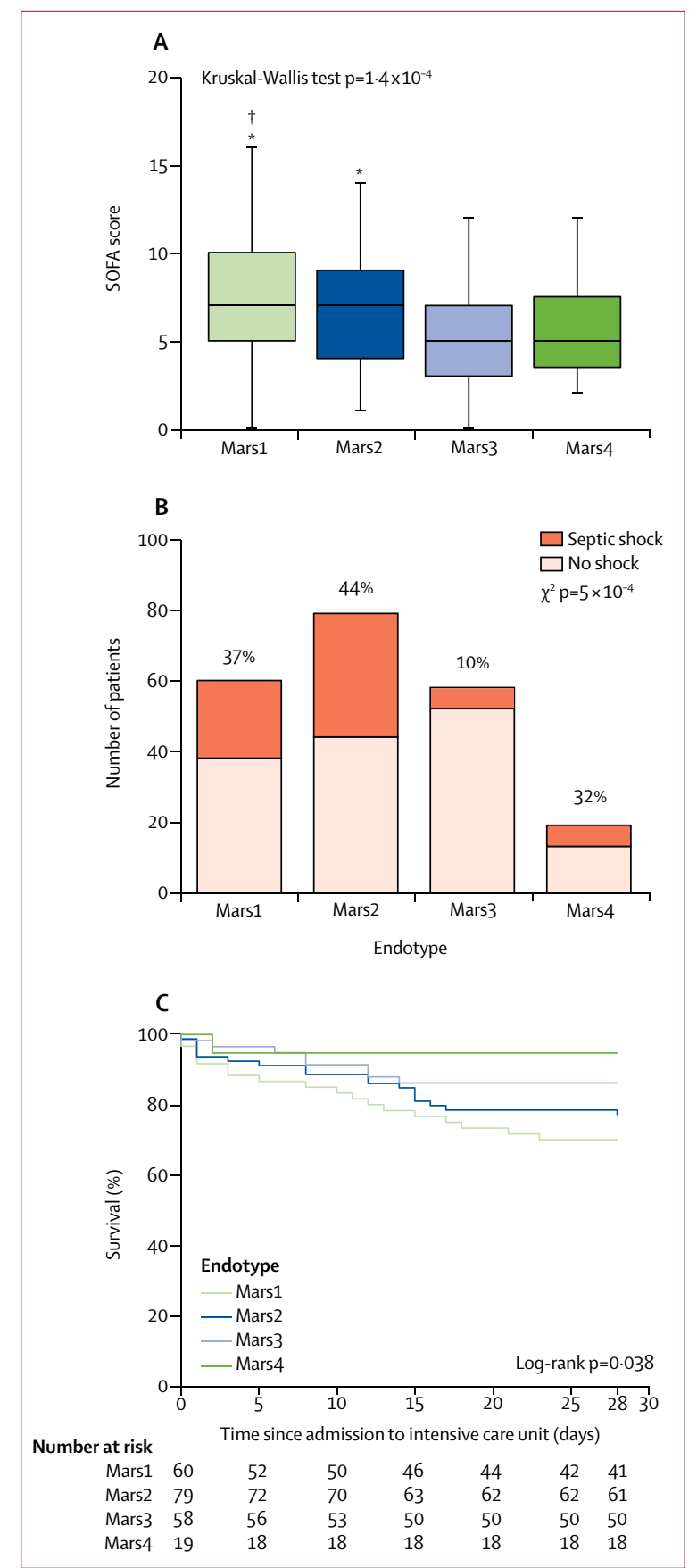

Figure 3: Assessment of sepsis molecular endotypes in the first validation cohort

Mars sepsis endotypes were assessed for the association with total SOFA scores (A; black horizontal line shows median score, top and bottom of box shows upper and lower quartiles, whiskers show extremes, and squares show outliers), septic shock ( $B$; the proportions of patients with septic shock within each endotype are shown at the top of each bar), and Kaplan-Meier survival analysis up to day 28 (C) SOFA $=$ sequential organ failure assessment. ${ }^{*} p<0.01$ vs Mars $3 .+p<0.05$ vs Mars4.

58 with Mars3 (HR 0.55 [0.25-1.17]; p=0.097), and one $(5 \%)$ of 19 with Mars4 (HR 0 21 [0.03-1.55]; $\mathrm{p}=0 \cdot 092$ ) had died. The multivariate HR (incorporating Charlson comorbidity indices) for death within 28 days of Mars1 classification equated to 1.91 (95\% CI $1 \cdot 05-3 \cdot 47$, $\mathrm{p}=0 \cdot 034)$. The association with mortality was also evident at 1-year follow-up (log-rank $\mathrm{p}=0 \cdot 0031)$, with Mars1classified patients having the worst outcome (appendix p 17). Combining clinical (APACHE IV) and molecular (sepsis endotype classification) data significantly improved 28-day mortality risk prediction (net reclassification improvement, continuous 0.38 [95\% CI $0 \cdot 01-0 \cdot 66], \mathrm{p}=0 \cdot 008$; integrated discrimination improvement $0 \cdot 028[0 \cdot 0018-0 \cdot 055], \mathrm{p}=0 \cdot 036$ ) compared with APACHE IV scores alone. The Hosmer-Lemeshow test showed proper model calibration (appendix p 17).

In the second validation cohort of patients with sepsis caused by community-acquired pneumonia $(n=265),{ }^{17}$ we also detected four endotypes (appendix $\mathrm{p}$ 18) with favourable stability. Consistent with the discovery and first validation cohort, SOFA scores, septic shock, and mortality were significantly associated with endotype (figure 4A-C). Patients with the Mars1 endotype had the worst prognosis, and 12 (34\%) of 35 Mars1 patients had died at 28 days (HR 2.02 [95\% CI 1.07-3.82]; $\mathrm{p}=0 \cdot 031$ ). Risk stratification by Mars2-4 was not fully consistent with the discovery cohort at 28 days: 26 (22\%) of 117 patients with the Mars2 endotype (HR 1 12 [0 - 66-1 .9]; $\mathrm{p}=0 \cdot 66), 13(13 \%)$ of 97 with Mars3 (HR 0.47 [0 25-0.88]; $\mathrm{p}=0.018$ ), and five $(31 \%)$ of 16 with Mars4 (HR 1.65 [0.66-4.13]; $\mathrm{p}=0 \cdot 29$ ) had died (figure 4C). No associations were seen between endotype and age, sex, or APACHE II score (appendix p 11).

Notably, APACHE IV scores were collected in the discovery and first validation cohort, whereas APACHE II scores were collected in the second validation cohort. Considering that the second validation cohort only consisted of patients with community-acquired pneumonia, and that Mars endotypes were associated with abdominal and lung site of infection in the discovery and first validation cohorts (appendix pp 7-10), we combined patients from the two all-cause sepsis cohorts (discovery and first validation cohort) to show that the four endotypes were present irrespective of the primary site of infection. For this analysis, we separately assessed the patients with the two most common causes of sepsis: pneumonia $(n=215)$ and peritonitis $(n=123)$. We detected four sepsis endotypes in both subgroups with favourable stability (appendix p 18); however, we detected different proportions of patients classified to each endotype A greater proportion of patients were classified as Mars3 or Mars4 in the pneumonia cohort than in the abdominal sepsis subgroup (appendix p 18). This suggests that although the four endotypes were clearly present in patients with both sites of infection, Mars3 and Mars4 endotypes might be more common in patients with pneumonia than in those with peritonitis.

In an exploratory cohort of paediatric patients with sepsis (GEO accession number GSE13904, $n=81{ }^{18}$ appendix p 12), we found three endotypes (Mars1, Mars2, 
and Mars4) with favourable stability (appendix p 19). In this cohort, Mars3 was not reliably detected and assessment of 28-day mortality and paediatric risk of mortality score ${ }^{31}$ showed no significant associations with sepsis endotype classification (appendix p 19).

To understand the biological underpinnings of the molecular endotypes we analysed the association of sepsis endotypes with leucocyte counts and differentials. Monocyte count was significantly associated with endotype in both the discovery and first validation cohorts (appendix pp 7-10), with a small overall effect size $\left(\eta^{2}\right)$ equating to $4 \cdot 3 \%$. Total leucocyte count was associated with sepsis endotypes only in the discovery cohort, whereas lymphocyte count was associated with endotypes only in the first validation cohort. No significant associations were found between neutrophil counts and endotypes.

Differential gene expression analysis and biological pathway inference were done for the four endotypes. Each endotype in the discovery cohort showed substantial alterations relative to the healthy cohort (figure 5A), with $77 \%$ of the gene expression responses common to all endotypes and Spearman's $\rho$ more than $0 \cdot 7$ (appendix p 20). Gene expression signatures specific to each endotype were also evident, most particularly in the high-risk Mars1 endotype (appendix p 20). The Mars1 endotype was characterised by a pronounced decrease in expression of genes corresponding to key innate and adaptive immune cell functions such as Toll-like receptor, nuclear factor $\kappa B$ (NFkB1) signalling, antigen presentation, and T-cell receptor signalling, concomitant with increased expression of specific metabolic pathway genes that included haem biosynthesis pathways (figure 5B; appendix p 20). The Mars2 endotype was characterised by increased expression of genes involved in pattern recognition, cytokine, cell growth, and mobility pathways, including NF- $\mathrm{kB}$, interleukin 6, inducible nitric oxide synthase, and $\mathrm{N}$-formyl-methionyl peptide signalling. The Mars4 endotype was also associated with increased expression of genes involved in pattern recognition and cytokine pathways, specifically interferon signalling, RIG1-like receptor and TREM1 signalling (figure 5B; appendix p 20). Finally, the Mars3 endotype was predominantly associated with increased expression of adaptive immune pathway genes, which included T-helper cell, natural killer cell, IL-4 signalling and B-cell development pathways (figure 5B; appendix $\mathrm{p}$ 20). The Mars 3 endotype, which was associated with a low risk compared with that of Mars1, showed a significant association with the previously described lowrisk sepsis response signature (SRS)2 group in the UK sepsis cohort (appendix p 20). ${ }^{17}$

To facilitate potential translation to clinical practice, we established sepsis endotype scores using a previously described and validated combinatorial approach., ${ }^{5,6}$ We assessed 77840 combinations of genes in the 140-gene classifier for classification of the four molecular endotypes and identified eight genes that, in specific combinations,

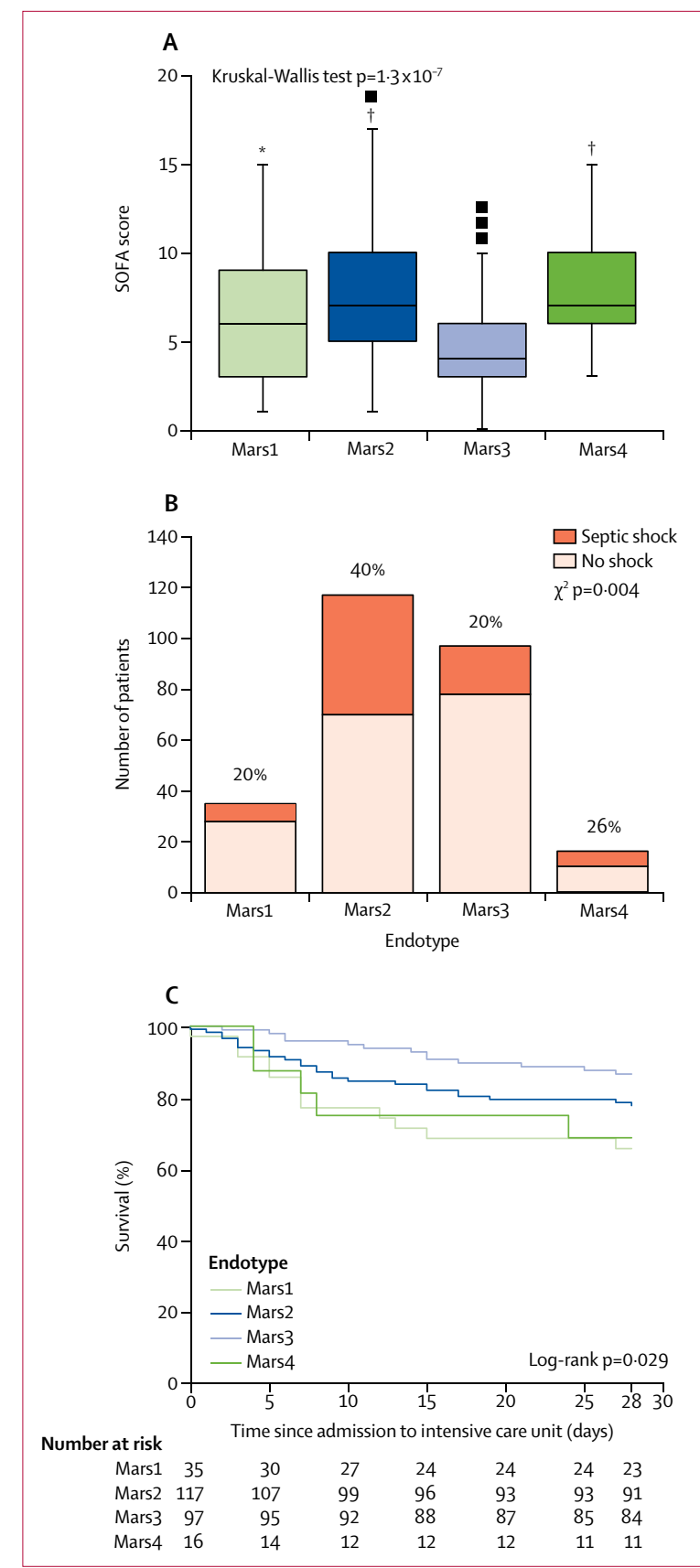

Figure 4: Assessment of sepsis molecular endotypes in the second validation cohort

Mars sepsis endotypes were assessed for the association with total SOFA scores (A; black horizontal line shows median score, top and bottom of box shows upper and lower quartiles, whiskers show extremes, and squares show outliers), septic shock ( $B$; the proportions of patients with septic shock within each endotype are shown at the top of each bar), and Kaplan-Meier survival analysis up to day 28 (C). SOFA=sequential organ failure assessment. * $p<0.05$ vs Mars3. $\dagger \mathrm{p}<0.001$ vs Mars3.

reliably stratified patients from the discovery cohort as sepsis molecular endotypes (figure 6). Gene expression ratios were used to classify patients as Mars1, 


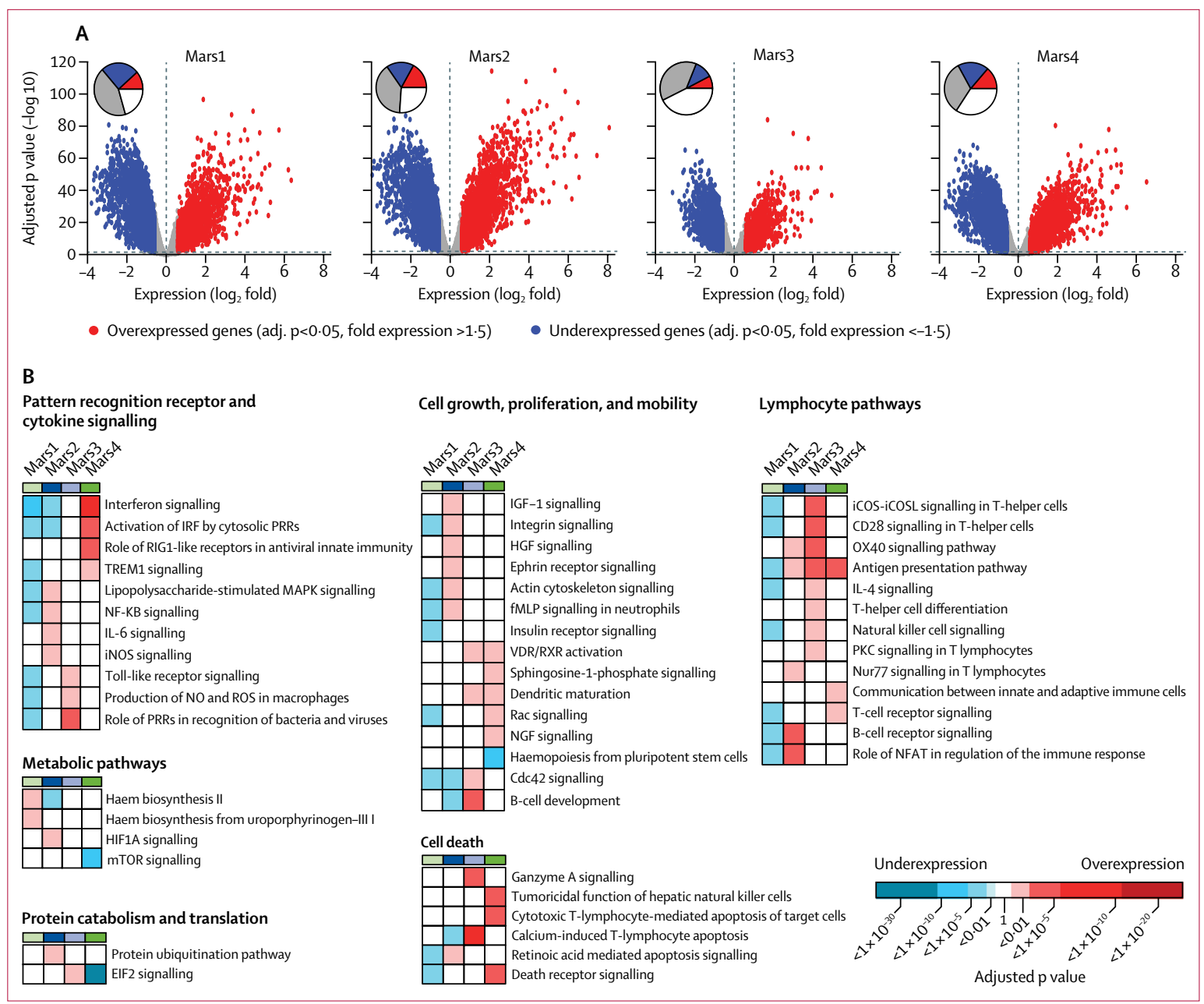

Figure 5: Biological interpretation of sepsis molecular endotypes

(A) Volcano plot of differential gene expression in the Mars1-4 groups of the discovery cohort compared with healthy individuals ( $n=42$ ). Plots integrate differential gene expression (discovery cohort $v$ s healthy participants; $x$-axis) and multiple-comparison adjusted $p$ values for endotypes versus healthy controls ( $y$-axis). Within plots, pie charts show the extent of gene expression changes: red slices show significantly overexpressed genes (adjusted p<0.05 and expression more than 1.5 times that of the healthy cohort), blue slices show significantly underexpressed genes (adjusted $p<0.05$ and expression more than 1.5-times decreased compared with the healthy cohort), and grey slices show significantly different gene expression (adjusted $p<0.05$ less than 1.5 -times increased or decreased compared with the healthy cohort). (B) Ingenuity pathway analysis of unique canonical signalling gene sets per endotype. Canonical signalling pathways were grouped into super pathways. Heatmaps show over-representation Fisher's test probabilities (considering multiple comparison adjusted $\mathrm{p}<0.01$ ).

bisphosphoglycerate mutase (BPGM): transporter 2, ATP binding cassette subfamily B member (TAP2); Mars2, growth arrest and DNA damage inducible alpha (GADD45A): polycomb group ring finger 5 (PCGF5); Mars3, AHNAK nucleoprotein (AHNAK): programmed cell death 10 (PDCD10); and Mars4, interferon induced protein with tetratricopeptide repeats 5 (IFIT5): glioma tumour suppressor candidate region gene 2 (GLTSCR2; also known as NOP53 ribosome biogenesis factor [NOP53]) endotypes (appendix p 21). These candidate biomarkers for Mars endotype membership also accurately classified patients in the two validation cohorts (appendix p 21). Collectively, these findings provide evidence for these gene combinations as candidate molecular biomarkers for the identification of sepsis molecular endotypes at ICU admission.

\section{Discussion}

We identified four endotypes (Mars1-4) in three heterogeneous cohorts of patients with sepsis on the basis of blood leucocyte genome-wide expression profiles at ICU admission. These sepsis endotypes had pathophysiological implications, and were not easily discernible by clinical characteristics. Common and distinct biological signatures characterised the four sepsis endotypes. The Mars1 endotype was consistently associated with the highest mortality, and the poor prognosis was associated with a notable decrease in expression of genes involved in innate and adaptive immune functions. By contrast, the relatively low-risk Mars3 endotype had increased expression of adaptive immune or T-cell functions. Eight genes were established as candidate biomarkers for the identification of sepsis endotypes at ICU admission, with BPGM and 
TAP2 transcripts denoting the Mars1 endotype. BPGM encodes a small molecule, 2,3-diphosphoglycerate, which binds to haemoglobin in red blood cells thereby decreasing the oxygen affinity of haemoglobin..$^{32}$ TAP2 is a member of the superfamily of ATP-binding cassette transporters involved in antigen presentation. ${ }^{33}$

Our results provide robustness to the classification of adults with poor-prognosis sepsis as Mars1 endotype. Risk stratification of patients as Mars2-4 might not be as clinically relevant for prognosis, especially the Mars4 endotype, which had variable mortality occurrence in the different cohorts. Furthermore, these findings suggest Mars1-4 endotype classification is only partially applicable to children with sepsis; only Mars 1, 2, and 4 groups are applicable to this population.

Improvements in the precision and breadth of omics data that can be observed in patients with sepsis have set the stage for sophisticated methods to better understand these sources of high-dimensional data, especially in relation to clinical traits. Through the use of consensusbased clustering techniques, important substructures in disease populations have been identified. ${ }^{10,11,19,21}$ An overarching observation of patient endotypes was their association with varying degrees of disease severity and mortality. Our findings showed a consistent association of the Mars1 sepsis endotype with high mortality across the cohorts. These results support those of previous studies $^{17,34}$ in patients with and without sepsis. The study by Maslove and colleagues ${ }^{34}$ investigated neutrophil transcriptional profiles from patients with and without sepsis and the UK GAinS study ${ }^{17}$ enrolled patients with sepsis due to community-acquired pneumonia, and both studies identified two sepsis endotypes with prognostic value. Our analysis of the GAinS cohort, applying an ensemble of methods for rigorously measuring quality and stability of sample partitioning, as well as classification by machine learning, showed that a fourendotype model was favourable in this cohort. Of note, the low-risk SRS2 endotype ${ }^{17}$ was highly correlated to the low-risk Mars3 sepsis endotype, both characterised by heightened expression of genes predominantly involved in adaptive immune functions (mainly $\mathrm{T}$ cell). The underdeveloped nature of adaptive immunity in children, ${ }^{35,36}$ possibly together with the high proportion of shock cases enrolled in the paediatric sepsis cohort we analysed, ${ }^{18}$ might explain, at least in part, the unstable classification of paediatric patients with sepsis to the Mars3 sepsis endotype.

Although we identified all four endotypes in patients with either pneumonia or peritonitis, Mars3 and Mars4 were much more common in patients with pneumonia than those with peritonitis. We did not establish a conclusive explanation for this finding. Surgical interventions directly before or after ICU admission, which are common in patients with peritonitis but not in those with pneumonia, could partially influence endotype classification.
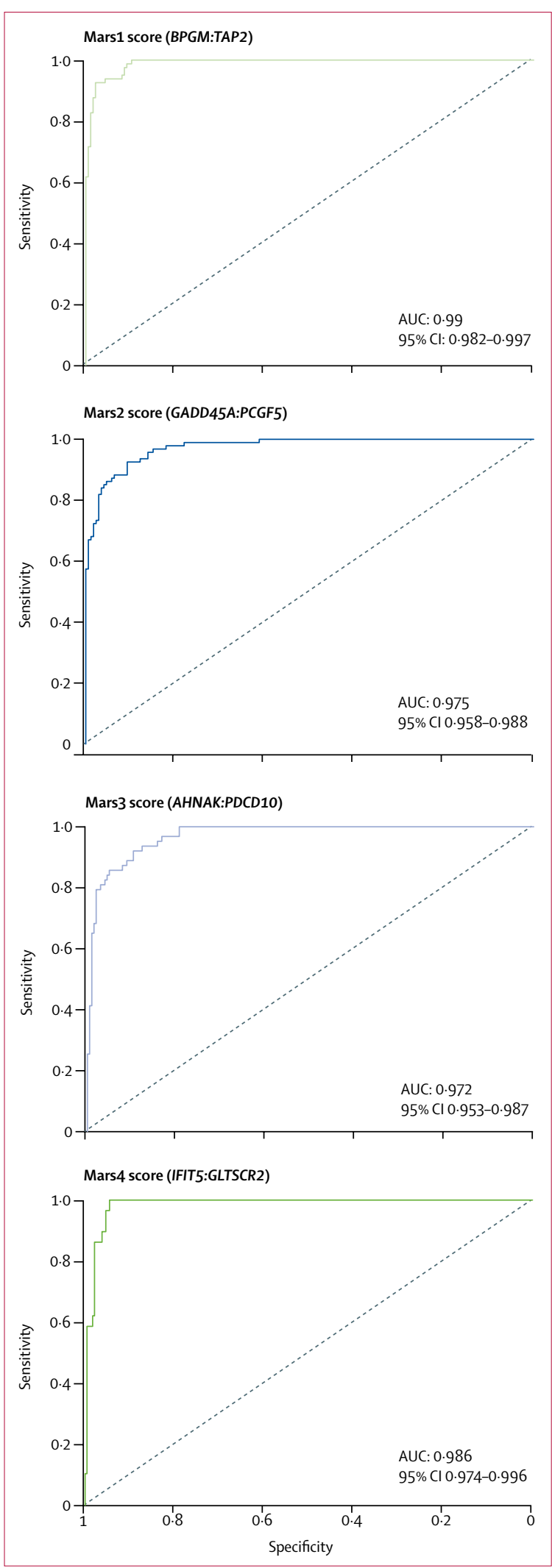

Figure 6: Establishment of candidate sepsis molecular endotype biomarkers in the discovery cohort Receiver operator characteristics AUC analyses of gene expression sepsis endotype classifier scores. $95 \% \mathrm{Cl}=$ bootstrap resampled $95 \% \mathrm{Cl}$. AUC=area under the curve. 
Our study has limitations. We enrolled only patients with an expected length of ICU stay longer than $24 \mathrm{~h}$, mainly to exclude elective (cardiopulmonary) surgical patients who routinely stay on the ICU from a few hours up to one night. Nonetheless, this selection might impair the generalisability of our findings. For the discovery and first validation cohorts, only patients with an infection likelihood of definite and probable were included. In the second validation cohort, infection likelihood was not scored, and inclusion of patients with an infection likelihood of possible in the discovery cohort might have yielded different results. The discovery cohort was admitted to a single ICU in the Netherlands, which could affect the generalisability of the results. We countered this by validating the results in two other cohorts from 30 different hospitals. The clinical value of the candidate endotype biomarkers was only assessed by receiver operating characteristic analysis using microarray gene expression data. Further analysis of the candidate biomarkers with other methods such as reverse transcription quantitative PCR in additional cohorts is warranted.

Classification of heterogeneous sepsis populations into molecular endotypes might in the future provide clues for targeted therapies for specific subgroups. The poor prognosis sepsis endotype, Mars1, was characterised by decreased expression in genes that function in both innate and adaptive immune mechanisms concomitant to high expression of specific cellular metabolic pathways, including haem biosynthesis. Glycine accumulation, biosynthesised by serine derived from the glycolysis pathway intermediate 3-phosphoglycerate, fuels haem biosynthesis and in turn modulates ATP synthesis via oxidative phosphorylation in mitochondria. ${ }^{37}$ Defects in immunometabolic circuits, including glycolysis and oxidative phosphorylation, have been shown to underlie immunoparalysis in sepsis. ${ }^{38}$ Therefore, these findings suggest that Mars1 might be an endotype that is characterised by immunoparalysis and poor prognosis. The Mars2 and Mars4 endotypes were characterised by high expression of genes involved in pro-inflammatory (eg, NF- $\mathrm{kB}$ signalling) and innate (eg, interferon signalling) immune reactions. Thus, Mars2 and Mars4 might be distinct hyperinflammatory endotypes. Genes with increased expression in the lowest-risk Mars3 endotype were mostly involved in adaptive immune or T-cell pathways, supporting the hypothesis that intact T-cell functions improve sepsis outcome. ${ }^{39}$

Clinical trials for sepsis seeking to modify the host immune response have thus far yielded no beneficial effect on outcome. ${ }^{40}$ A growing body of evidence supports the reassessment of clinical trial designs ${ }^{41}$ to include biomarkers reflecting the status of the host response..$^{40,42}$ We envisage that endotype classification might provide more homogeneity to the notoriously heterogeneous population of patients with sepsis: the molecular endotypes described here show that sepsis presents a heterogeneous syndrome with distinct pathophysiological profiles in patients that are not clinically distinguishable. By deriving two-gene biomarkers for each endotype we provided evidence that molecular subtyping of patients with sepsis (using rapid bedside PCR-based tests) is feasible in clinical practice, and the technology to produce such tests with automated generation of results within several hours already exists. Future research is required to identify targetable pathways within these endotypes that could be modulated as part of personalised therapies in subgroups of patients with sepsis. This research should involve prospective validation and longitudinal analyses of biomarkers during the course of the disease to establish whether patients can switch endotypes. The next step for implementation of personalised medicine in clinical sepsis management is to combine the measurement of a biomarker set that provides insight into the activity of an immunological pathway with a specific intervention targeting that pathway. This approach, which has been named theranostics, would allow the use of molecular biomarkers both for selection of patients for a specific therapy and for monitoring thereof.

\section{Contributors}

BPS and TvdP conceived the study and design. BPS, LAvV, and MAW did the laboratory work. BPS, LAvV, and AHZ contributed to the statistical analysis. EED, KLB, PN, MJS, JH, OLC, MJB, CJH, HRW, and JCK provided administrative, technical, or material support. BPS and TvdP drafted the paper. TvdP and MJB obtained funding. BPS and TvdP led the study.

\section{Declaration of interests}

BPS and TvdP report a patent pending for a molecular biomarker for prognosis of sepsis patients (ref: 2016-054EP PR). All other authors declare no competing interests.

\section{Acknowledgments}

We would like to thank all members of the MARS consortium (listed in the appendix pp 4-5) for the participation in data collection and Marek Franitza and Mohammed R Toliat (Cologne Center for Genomics, University of Cologne, Cologne, Germany) for microarray data collection. We would also like to thank all patients and volunteers who participated in the study.

\section{References}

1 Angus DC, van der Poll T. Severe sepsis and septic shock. $N$ Engl J Med 2013; 369: 840-51.

2 Liu V, Escobar GJ, Greene JD, et al. Hospital deaths in patients with sepsis from 2 independent cohorts. JAMA 2014; 312: 90-92.

3 Cohen J, Vincent JL, Adhikari NK, et al. Sepsis: a roadmap for future research. Lancet Infect Dis 2015; 15: 581-614.

4 Maslove DM, Wong HR. Gene expression profiling in sepsis: timing, tissue, and translational considerations. Trends Mol Med 2014; 20: 204-13.

5 Scicluna BP, Klein Klouwenberg PM, van Vught LA, et al. A molecular biomarker to diagnose community-acquired pneumonia on intensive care unit admission. Am J Respir Crit Care Med 2015; 192: 826-35.

6 McHugh L, Seldon TA, Brandon RA, et al. A molecular host response assay to discriminate between sepsis and infection-negative systemic inflammation in critically ill patients: discovery and validation in independent cohorts. PLoS Med 2015; 12: e1001916.

7 Sweeney TE, Shidham A, Wong HR, Khatri P. A comprehensive time-course-based multicohort analysis of sepsis and sterile inflammation reveals a robust diagnostic gene set. Sci Transl Med 2015; 7: 287ra71. 
8 Parlato M, Cavaillon JM. Host response biomarkers in the diagnosis of sepsis: a general overview. Methods Mol Biol 2015; 1237: 149-211.

9 Sweeney TE, Wong HR. Risk stratification and prognosis in sepsis: what have we learned from microarrays? Clin Chest Med 2016; 37: 209-18.

10 Curtis C, Shah SP, Chin SF, et al. The genomic and transcriptomic architecture of 2,000 breast tumours reveals novel subgroups. Nature 2012; 486: 346-52.

11 Guinney J, Dienstmann R, Wang X, et al. The consensus molecular subtypes of colorectal cancer. Nat Med 2015; 21: 1350-56.

12 Klein Klouwenberg PM, Ong DSY, Bos LDJ, et al. Interobserver agreement of centers for disease control and prevention criteria for classifying infections in critically ill patients. Crit Care Med 2013; 41: 2373-78.

13 van Vught LA, Klein Klouwenberg PM, Spitoni C, et al. Incidence, risk factors, and attributable mortality of secondary infections in the intensive care unit after admission for sepsis. JAMA 2016; 315: 1469-79.

14 Garner JS, Jarvis WR, Emori TG, Horan TC, Hughes JM. CDC definitions for nosocomial infections, 1988. Am J Infect Control 1988; 16: $128-40$

15 Calandra T, Cohen J. The international sepsis forum consensus conference on definitions of infection in the intensive care unit. Crit Care Med 2005; 33: 1538-48.

16 Levy MM, Fink MP, Marshall JC, et al. 2001 SCCM/ESICM/ACCP/ ATS/SIS international sepsis definitions conference. Crit Care Med 2003; 31: 1250-56.

17 Davenport EE, Burnham KL, Radhakrishnan J, et al. Genomic landscape of the individual host response and outcomes in sepsis: a prospective cohort study. Lancet Respir Med 2016; 4: 259-71.

18 Wong HR, Cvijanovich N, Allen GL, et al. Genomic expression profiling across the pediatric systemic inflammatory response syndrome, sepsis, and septic shock spectrum. Crit Care Med 2009; 37: $1558-66$.

19 Verhaak RG, Hoadley KA, Purdom E, et al. Integrated genomic analysis identifies clinically relevant subtypes of glioblastoma characterized by abnormalities in PDGFRA, IDH1, EGFR, and NF1. Cancer Cell 2010; 17: 98-110.

20 Wilkerson MD, Hayes DN. ConsensusClusterPlus: a class discovery tool with confidence assessments and item tracking. Bioinformatics 2010; 26: 1572-73.

21 De Sousa E Melo F, Wang X, Jansen M, et al. Poor-prognosis colon cancer is defined by a molecularly distinct subtype and develops from serrated precursor lesions. Nat Med 2013; 19: 614-18.

22 Rousseeuw PJ. Silhouettes: a graphical aid to the interpretation and validation of cluster analysis. Comput Appl Math 1987; 20: 53-65.

23 Maechler M, Rousseeuw P, Struyf A, Hubert M, Hornik K. Cluster: cluster analysis basics and extensions. R package, version 1153. 2014

24 Brunet JP, Tamayo P, Golub TR, Mesirov JP. Metagenes and molecular pattern discovery using matrix factorization. Proc Natl Acad Sci USA 2004; 101: 4164-69.
25 Breiman L. Random forests. Machine Learning 2001; 45: 5-32.

26 Slawski M, Daumer M, Boulesteix AL. CMA: a comprehensive Bioconductor package for supervised classification with high dimensional data. BMC Bioinformatics 2008; 9: 439.

27 Kundu S, Aulchenko YS, van Duijn CM, Janssens AC. PredictABEL: an $\mathrm{R}$ package for the assessment of risk prediction models. Eur J Epidemiol 2011; 26: 261-64.

28 Zimmerman JE, Kramer AA, McNair DS, Malila FM. Acute Physiology and Chronic Health Evaluation (APACHE) IV: hospital mortality assessment for today's critically ill patients. Crit Care Med 2006; 34: 1297-310.

29 Vincent JL, Moreno R, Takala J, et al. The SOFA (Sepsis-related Organ Failure Assessment) score to describe organ dysfunction/ failure. Intensive Care Med 1996; 22: 707-10.

30 Pencina MJ, D'Agos'ino RB Sr, D’Agostino RB Jr, Vasan RS. Evaluating the added predictive ability of a new marker: from area under the ROC curve to reclassification and beyond. Stat Med 2008; 27: 157-72; discussion 207-12.

31 Pollack MM, Ruttimann UE, Getson PR. Pediatric risk of mortality (PRISM) score. Crit Care Med 1988; 16: 1110-16.

32 Rosa R, Blouquit Y, Calvin MC, Prome D, Prome JC, Rosa J. Isolation, characterization, and structure of a mutant 89 Arg - Cys bisphosphoglycerate mutase. Implication of the active site in the mutation. J Biol Chem 1989; 264: 7837-43.

33 Kelly A, Powis SH, Kerr LA, et al. Assembly and function of the two $\mathrm{ABC}$ transporter proteins encoded in the human major histocompatibility complex. Nature 1992; 355: 641-44.

34 Maslove DM, Tang BM, McLean AS. Identification of sepsis subtypes in critically ill adults using gene expression profiling. Crit Care 2012; 16: R183.

35 Wheeler DS, Wong HR, Zingarelli B. Pediatric sepsis-part I: "Children are not small adults!". Open Inflamm J 2011; 4: 4-15.

36 PrabhuDas M, Adkins B, Gans H, et al. Challenges in infant immunity: implications for responses to infection and vaccines. Nat Immunol 2011; 12: 189-94.

37 Amelio I, Cutruzzola F, Antonov A, Agostini M, Melino G. Serine and glycine metabolism in cancer. Trends Biochemical Sci 2014; 39: 191-98.

38 Cheng SC, Scicluna BP, Arts RJ, et al. Broad defects in the energ metabolism of leukocytes underlie immunoparalysis in sepsis. Nat Immunol 2016; 17: 406-13.

39 Hotchkiss RS, Monneret G, Payen D. Sepsis-induced immunosuppression: from cellular dysfunctions to immunotherapy. Nat Rev Immunol 2013; 13: 862-74.

40 Marshall JC. Why have clinical trials in sepsis failed? Trends Mol Med 2014; 20: 195-203.

41 Angus DC. The search for effective therapy for sepsis: back to the drawing board? JAMA 2011; 306: 2614-15.

42 van der Poll T. Future of sepsis therapies. Crit Care 2016; 20: 106. 